\title{
An Enhanced Crack-Plugging and Pressure-Bearing Oil-Based Drilling Fluid System
}

\author{
Liming Dong
}

Technology Development Department of SINOPEC Shengli Oilfield Service Corporation, Dongying 257100, China

Corresponding Author Email: dongliming.ossl@ sinopec.com

https://doi.org/10.18280/eesrj.070406

Received: 14 September 2020

Accepted: 27 November 2020

\section{Keywords:}

oil-based drilling fluid (OBDF), leakageproof, crack plugging, pressure-bearing, strong-force chain network, leakage loss, filtration loss, permeability plugging apparatus (PPA)

\begin{abstract}
Compared with water-based drilling fluids (WBDF), oil-based drilling fluids (OBDF) are more prone to borehole leakage loss. Once borehole leakage occurs, it's difficult to handle the leakage site and it will inevitably cause great economic losses. To cope with the major difficulties in OBDF leakage-proof and crack plugging, this paper conducted experiments on an enhanced crack-plugging and pressure-bearing OBDF system starting from the borehole leakage prevention measures. Then, by selecting and optimizing the materials of leakage-proof agent and crack-plugging agent for OBDF, this study prepared a newtype OBDF drilling leakage-proof agent and a new-type OBDF still crack-plugging agent and discussed their action mechanisms. Laboratory experiment and mechanism analysis results showed that, a well-proportioned combination of different type OBDF leakageproof and crack-plugging materials such as rigid bridging particles, elastic filling particles, and fiber materials had a synergistic plugging effect and quickly formed a dense crack-plugging and pressure-bearing layer with a stable network structure (the "strongforce chain network" model), which had significantly improved the crack-plugging and pressure-bearing performance of the OBDF, and further optimized the enhanced crackplugging and pressure-bearing OBDF system; before and after the heated rolling aging, the rheological properties and filtration loss performance of the system were good, and the filtration loss of the sand filter cake of the Permeability Plugging Apparatus (PPA) was only $11.4 \mathrm{~mL}$, therefore, the proposed agents exhibited good performance in drilling leakage-proof, crack-plugging, and pressure-bearing, and they were helpful to improve the pressure bearing ability of the earth stratums.
\end{abstract}

\section{INTRODUCTION}

With the continuous exploration of oil and gas resources, conventional oil and gas resources are on the decrease and the drilling exploration scope has extended to deep and ultra-deep earth stratums, deep sea and ocean, and pressure-depleted reservoir stratums. The geological conditions of these stratums are very complicated, which have proposed higher requirements for the drilling fluid systems. Under such harsh environment, oil-based drilling fluids (OBDF) have become the most preferred drilling fluid system, but it has very serious leakage loss problem, and often there are other complex drilling situations such as circulation loss, leakage-overflow co-existence, and borehole wall collapse, etc., which have greatly limited the large-scale application of OBDF $[1,2]$.

Aiming at the OBDF leakage loss problem, scholars have conducted various theoretical and experimental research works. For example, Onyia [3] found that, regardless of WBDF or OBDF, the initial cracking pressure of the earth stratum is basically the same, but in case of OBDF, the crack extension pressure and the crack reopening pressure are much lower than those in case of WBDF (Figure 1), and this is because the OBDF mud cake cannot achieve crack tip pressure isolation. Dupriest [4] and Benaissa et al. [5] further pointed out that about $90 \%$ of the drilling fluid leakage loss problems are caused by the extension and expansion of natural or induced cracks, and the low crack extension pressure is the root cause of the serious OBDF leakage loss problem.
Aderibigbe and Lane [6] carried out a series of mechanical tests and evaluation experiments, and the research results showed that, once the OBDF infiltrates into the stratums along the micro-cracks or through the connected pores, its surfactant components such as emulsifying agent or wetting agent would contact the surface of the rock in the stratums and change the properties of the rock surface, resulting in decrease in the tensile and shear strength of the rock, and it can easily induce rock cracks and lead to large-scale drilling fluid leakage loss. For this reason, various OBDF crack plugging materials such as thermosetting rubber, oil-absorbing swelling resin, oilbased gel, and composite plugging agent have been developed [7-9], and related field tests have been carried out; however, for now, there isn't a set of efficient OBDF leakage-proof and plugging techniques or complete supporting measures.

Above research results show that, compared with WBDF, OBDF is more prone to wellbore leakage loss, and it's very difficult to cope with the leakage site. Now, passive plugging technique is still the main measure for dealing with such leakage, and once borehole leakage occurs, it will inevitably cause great economic losses. Starting from an analysis on the mechanism of OBDF leakage and the leakage prevention techniques and measures, this paper conducted experiments on the techniques of borehole wall strengthening and crack plugging for OBDF; by selecting and optimizing the materials of leakage-proof agent and crack plugging agent for OBDF, this study prepared a new-type OBDF drilling leakage-proof agent and a new-type OBDF still crack plugging agent and 
discussed their action mechanisms. Finally, this paper proposed an enhanced crack-plugging and pressure-bearing OBDF system.

\section{EXPERIMENTS ON OBDF DRILLING LEAKAGE PREVENTION TECHNIQUES}

\subsection{A new-type OBDF drilling leakage-proof agent}

In recent years, the sand filter cake Permeability Plugging Test (PPT) has become an important method to evaluate the leakage-proof and plugging performance of drilling fluids. This method measures the filtration (leakage) loss of a drilling fluid to evaluate its leakage-proof and plugging performance by simulating the downhole high-temperature and highpressure environment, and taking sand filter cakes with different permeability values (such as $20 \mu \mathrm{m}^{2}, 10 \mu \mathrm{m}^{2}, 2 \mu \mathrm{m}^{2}$ ) as infiltration medium $[10,11]$.

After comprehensively considering the current plugging theories and the particle size distribution of leak-proof and plugging materials, a sand filter cake with a permeability of $10 \mu \mathrm{m}^{2}$ was taken as the infiltration medium in the PPT, and various materials such as rigid bridging particles, elastic filling particles, high-strength microfibers, and softened particles were selected as drilling fluid leakage-proof and plugging materials. Based on the ideal filling theory, the total amount of all materials was kept at 5\%, then the addition amount of each material was adjusted to determine the optimal formula combination of the materials for the new-type OBDF drilling leakage-proof agent [12].

Through single-agent optimization, a set of OBDF basemud formula was selected as: $5 \#$ white oil $+2 \%$ emulsifying agent $+3 \%$ wetting agent $+0.5 \%$ thickening agent $+4 \%$ organic soil $+1 \%$ calcium oxide (barite weighted to $1.2 \mathrm{~g} / \mathrm{cm}^{3}$ ) . Experimental formula $1 \# \sim 6 \#$ shown in Table 1 was respectively added into the OBDF base-mud, after heated rolling at $120^{\circ} \mathrm{C}$ for $16 \mathrm{~h}$, the optimal experimental formula was determined by PPT. According to Figure 1, after the drilling leakage-proof agents with different formulas were added, the PPA sand filter cake filtration loss of the OBDF had been significantly reduced; that is, the drilling leakage-proof performance of OBDF had been improved. Moreover, the 1\# experimental formula had the optimal effect, the PPA sand filter cake filtration loss was only $12.1 \mathrm{~mL}$.

Figure 2 is the curve of the cumulative percentage of the particle diameter $-\mathrm{D}^{1 / 2}$ of the leakage-proof and plugging materials in the above experiment. According to the figure, the curve of $1 \#$ experimental formula had the highest overlap with the baseline, so its plugging effect was the best. The curves of other experimental formulas all showed significant deviations from the baseline. Therefore, $1 \#$ experimental formula was selected as the optimal formula of the new-type OBDF drilling leakage-proof agent.
The new-type OBDF drilling leakage-proof agent is composed of rigid bridging particles, elastic filling particles, microfibers, and softening particles according to a certain particle size gradation. Different materials act their respective roles in the OBDF drilling leakage-proof agent and they have a synergistic plugging effect. The particles squeeze each other and are tightly combined to form a steady "strong-force chain network" plugging layer in the micro cracks and pores, thereby improving the plugging and pressure-bearing ability. According to the evaluation of PPT and the action mechanism analysis we can see that, the rigid bridging particles, elastic filling particles, microfibers, and softening particles quickly formed a dense plugging layer with a stable network structure (Figure 3), which had greatly reduced the PPA sand filter cake filtration loss from $58 \mathrm{~mL}$ to $12.1 \mathrm{~mL}$, and the drilling leakageproof performance of the OBDF had been significantly improved.

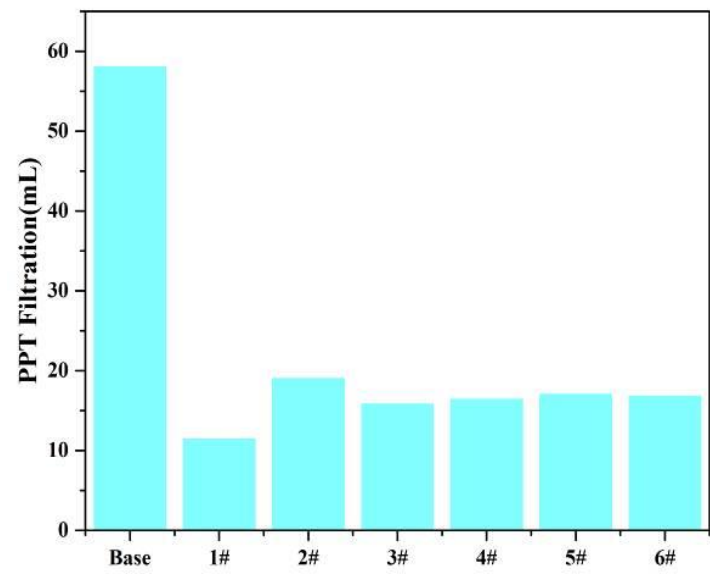

Figure 1. Comparison of PPA sand filter cake filtration loss $\left(120^{\circ} \mathrm{C} / 7.0 \mathrm{MPa}\right)$

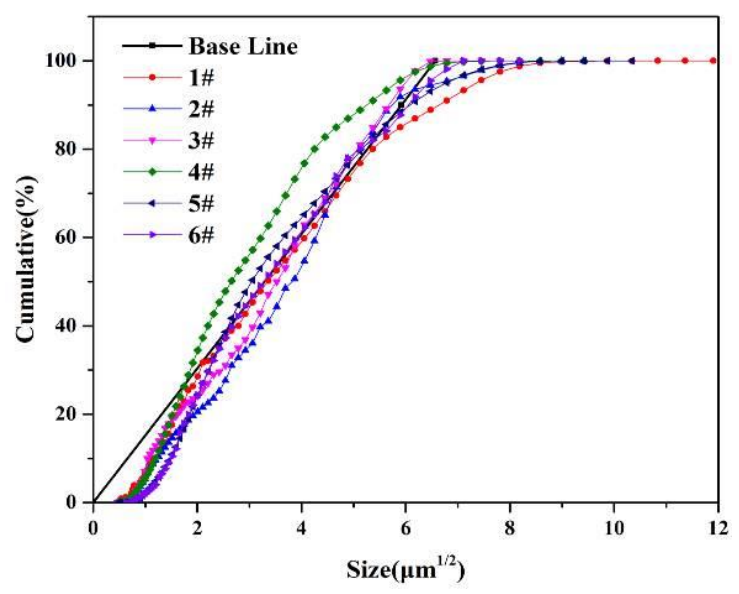

Figure 2. Curve of cumulative percentage of particle diameter- $\mathrm{D}^{1 / 2}$

Table 1. Experimental formula of the new-type OBDF drilling leakage-proof agent

\begin{tabular}{ccccc}
\hline Formula & $\begin{array}{c}\text { Rigid particles addition } \\
\text { amount } / \%\end{array}$ & $\begin{array}{c}\text { Elastic particles addition } \\
\text { amount } / \%\end{array}$ & $\begin{array}{c}\text { Microfibers addition } \\
\text { amount } / \%\end{array}$ & $\begin{array}{c}\text { Softened particles addition } \\
\text { amount } / \%\end{array}$ \\
\hline $1 \#$ & 1.0 & 0.5 & 2.5 & 1.0 \\
$2 \#$ & 1.0 & 0.5 & 3.0 & 0.5 \\
$3 \#$ & 1.0 & 1.0 & 2.0 & 1.0 \\
$4 \#$ & 1.5 & 0.5 & 2.0 & 1.0 \\
$5 \#$ & 1.5 & 1.0 & 2.0 & 0.5 \\
$6 \#$ & 2.0 & 0.5 & 1.5 & 1.0 \\
\hline
\end{tabular}



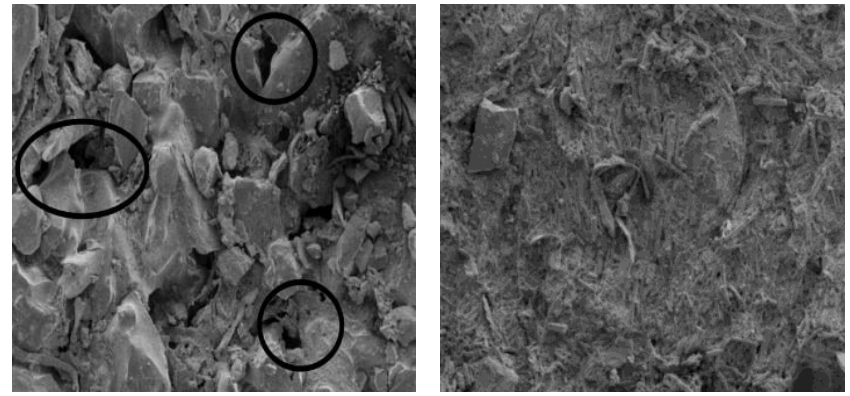

Figure 3. Scanning electron micrographs of the sand filter cake before and after the experiment (left-before, right-after)

\subsection{The enhanced crack plugging OBDF system}

Through the single-agent optimization experiment, key agents such as emulsifying agent, wetting agent, thickening agent and filtration loss reducing agent were selected and optimized to form a basic experimental formula of OBDF. Then, by adding the proposed new-type OBDF drilling leakage-proof agent, finally, the enhanced crack plugging OBDF formula was formed, and its rheological properties, filtration loss, and plugging performance were evaluated. The specific formula is: $5 \#$ white oil $+2.0 \%$ emulsify agent $+3.0 \%$ wetting agent $+0.5 \%$ thickening agent $+1.5 \%$ shear strength improving agent $+4.0 \%$ organic soil $+3.0 \%$ filtration loss reducing agent $+1.0 \%$ calcium oxide $+5.0 \%$ new-type OBDF drilling leakage-proof agent (barite weighted to $1.2 \mathrm{~g} / \mathrm{cm}^{3}$ ).

The test results of rheological properties and filtration loss of the OBDF before and after heated rolling at $120^{\circ} \mathrm{C}$ for $16 \mathrm{~h}$ are shown in Table 2. The rheological properties and filtration loss performance of the enhanced crack plugging OBDF system were relatively ideal. The emulsion stability before and after heated rolling was good, and completely satisfied the field requirements; the adding of the proposed new-type drilling leakage-proof agent will not significantly affect the rheology properties and filtration loss performance of the OBDF.

Sand filter cakes with a permeability of $10 \mu \mathrm{m}^{2}$ were taken as the filtration medium, and the PPT was conducted to evaluate the leakage-proof and plugging performance of the basic experimental formula of OBDF and the proposed enhanced crack plugging OBDF formula. According to Figures 4 and 5, the filtration loss of the PPA sand filter cake is linearly related to the square root of time, the intercept of the straight line on the $y$-axis is the instantaneous filtration loss, and the slope of the straight line is the static filtration loss rate.
Compared with the basic experimental formula, due to the adding of the proposed new-type drilling leakage-proof agent, the enhanced crack plugging OBDF system has good crack plugging and pressure-bearing ability. The PPA sand filter cake filtration loss was decreased from $30 \mathrm{~mL}$ to $11.4 \mathrm{~mL}$; the instantaneous filtration loss and the static filtration loss rate was only $2.61 \mathrm{~mL}$ and $0.54 \mathrm{~mL} / \mathrm{min}^{1 / 2}$, respectively.

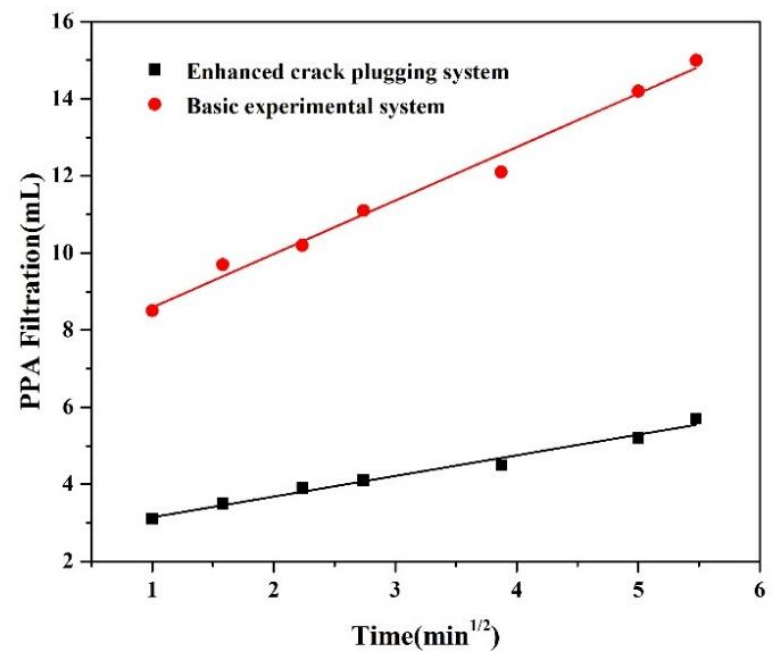

Figure 4. Relationship between PPA sand filter cake filtration loss and time $\left(\mathrm{min}^{1 / 2}\right)$

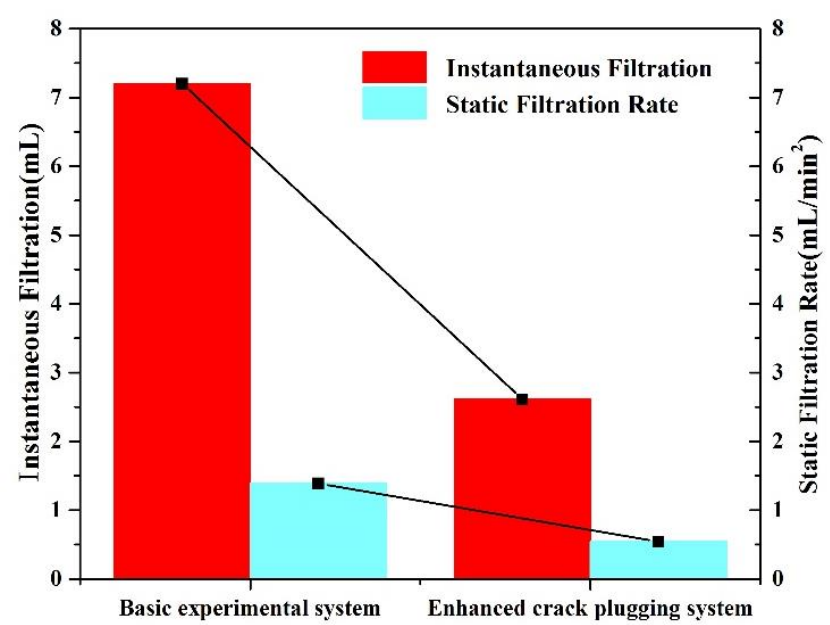

Figure 5. Comparison of instantaneous filtration loss and static filtration loss rate

Table 2. Rheological properties and filtration loss performance of the enhanced crack plugging OBDF system

\begin{tabular}{|c|c|c|c|c|c|c|c|}
\hline Formula & Condition & $\mathrm{AV} / \mathrm{mPa} \cdot \mathrm{s}$ & $\mathrm{PV} / \mathrm{mPa} \cdot \mathrm{s}$ & $\mathrm{YP} / \mathrm{Pa}$ & $\mathrm{Gel} / \mathrm{Pa} / \mathrm{Pa}$ & $\mathrm{ES} / \mathrm{V}$ & FLнтнр/mL \\
\hline \multirow{2}{*}{ Basic formula } & Before heated rolling & 46.0 & 37.0 & 9.0 & $8.5 / 11.5$ & 1980 & \multirow{2}{*}{6.1} \\
\hline & After heated rolling & 48.0 & 39.0 & 9.0 & $9.5 / 11.0$ & 1530 & \\
\hline \multirow{2}{*}{ Enhanced crack plugging OBDF formula } & Before heated rolling & 49.0 & 38.0 & 11.0 & $9.5 / 13.0$ & 1810 & \multirow{2}{*}{6.0} \\
\hline & After heated rolling & 48.0 & 38.0 & 10.0 & $10.0 / 11.5$ & 1568 & \\
\hline
\end{tabular}

Note: The rheological properties of OBDF were tested at $50^{\circ} \mathrm{C}$.

\section{EXPERIMENTS ON OBDF STILL CRACK PLUGGING TECHNIQUES}

During the drilling process, due to the changes in the in-situ stress in the area near the borehole wall, the stratum crack opening often extends from the micron level to the millimeter level, and the crack opening changes dynamically under stress disturbance, which can easily cause serious drilling fluid leakage loss problem [13]. At this time, due to the large leakage channel and fast leakage rate, it is difficult to form a dense plugging layer with a stable structure near the cracks. The on-site drilling fluid plugging operation is quite difficult, 
and it usually causes great economic losses. Therefore, for the millimeter-level cracks OBDF leakage loss problem, it is necessary to further optimize the composition and structure of the plugging and pressure-bearing layer, and conduct experiments on OBDF still crack plugging techniques.

\subsection{The new-type OBDF still crack plugging agent}

With cracks with an opening of $1 \mathrm{~mm}$ and rigid bridging particles, elastic filling particles, and fiber materials as OBDF leakage-proof and plugging materials, this study developed a new-type OBDF still crack plugging agent and conducted experiments on it, also, an ideal crack plugging and pressurebearing layer model was constructed and its action mechanism was discussed.

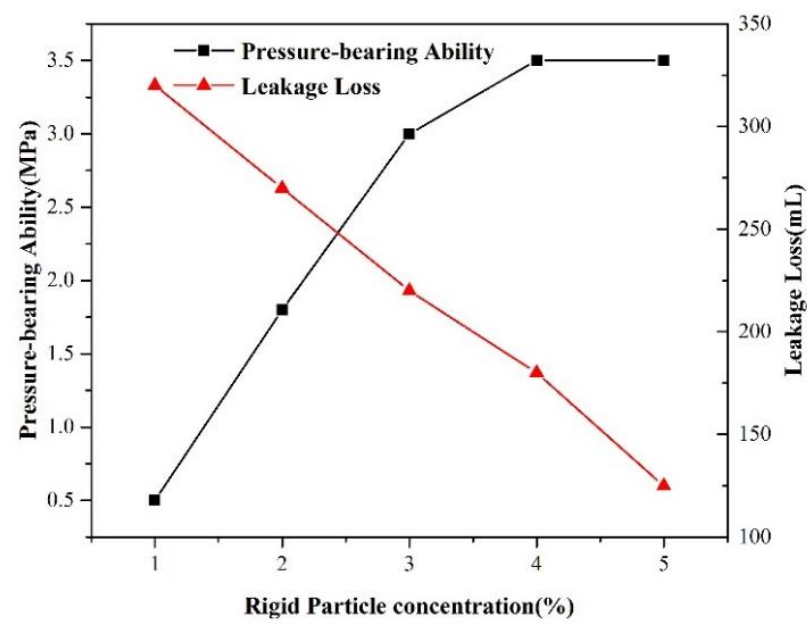

Figure 6. Experimental results of crack plugging with rigid particles

In the experiment, calcium carbonate particles (with a particle size of $0.6 \sim 1.0 \mathrm{~mm}$ ) were taken as the rigid bridging particles, then rigid bridging particles of different concentrations were added into the OBDF base mud to carry out the crack plugging experiment, and the experimental results are shown in Figure 6. First, with the increase of the bridging particle concentration, the crack plugging and pressure-bearing ability increased as well; when the particle concentration was $5 \%$, the crack plugging and pressurebearing ability reached a maximum value of only $3.5 \mathrm{MPa}$, and the crack leakage loss was as high as $125 \mathrm{~mL}$. Then, based on the " $4 \%$ bridging particle concentration", elastic filling particles of different concentrations were added; according to Figure 7, as the concentration of elastic particles increased, the crack plugging and pressure-bearing ability increased rapidly, when the elastic particle concentration was $2 \%$, the crack plugging and pressure-bearing ability reached $6.5 \mathrm{MPa}$, but the crack leakage loss was still relatively large $(75 \mathrm{~mL})$. At last, based on the " $4 \%$ bridging particle concentration and $2 \%$ elastic particle concentration", fiber materials were added to further optimize the composition and structure of the crack plugging and pressure-bearing layer; according to Figure 8, adding $1 \%$ fiber material can significantly increase the crack plugging ability to $10 \mathrm{MPa}$ (the maximum experimental pressure of the device) and greatly reduce the leakage loss. Through the above-mentioned experiment, the new-type OBDF still crack plugging agent was developed, and its formula is: $4 \%$ rigid bridging particles $+2 \%$ elastic filling particles $+1 \%$ fiber material .

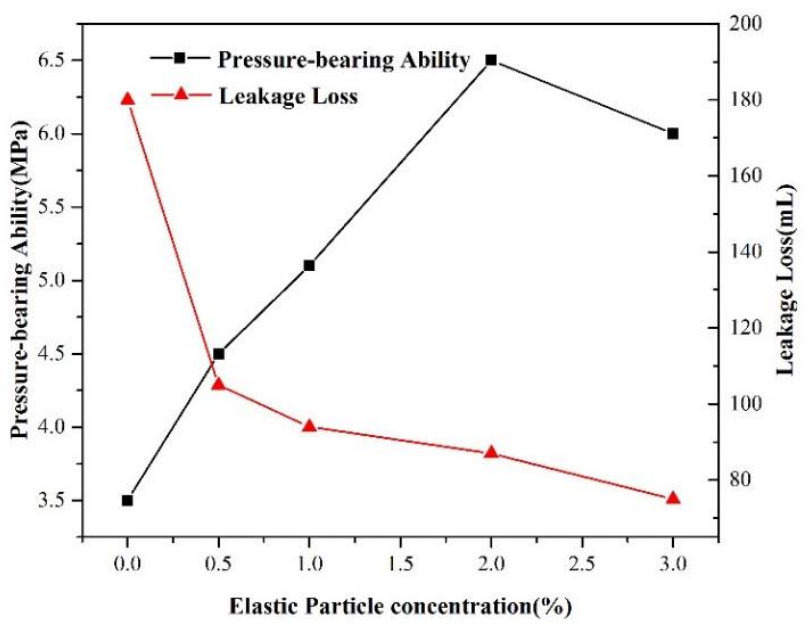

Figure 7. Experimental results of crack plugging with rigid and elastic particles

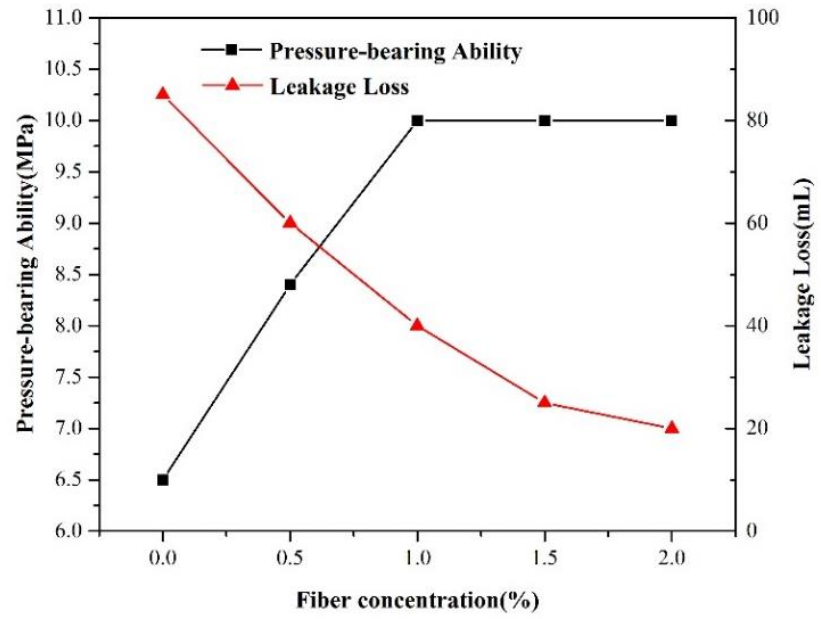

Figure 8. Experimental results of crack plugging with rigid and elastic particles and fibers

\subsection{Action mechanism of the new-type OBDF still crack plugging agent}

According to the basic mechanic principles of particulate materials, the crack-plugging pressure-bearing layer is a typical particulate material system composed of different types of drilling fluid leak-proof and plugging materials. It has the characteristics of multi-scale structures such as the single particle micro scale, the "force chain network" meso scale, and the plugging layer macro scale; wherein the "force chain network" constituted based on the micro scale contact forces controls the macro mechanical properties of the particulate matter system.

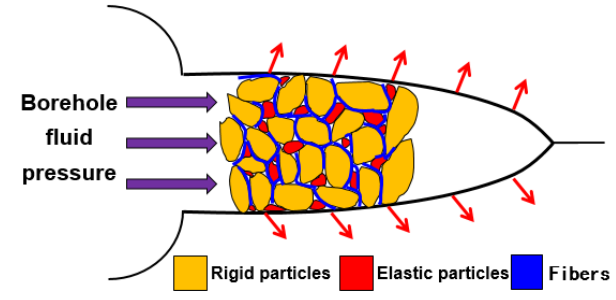

Figure 9. Model of the ideal crack-plugging pressure-bearing layer 
The new-type OBDF still crack-plugging agent is composed of rigid bridging particles, elastic filling particles, fiber materials and other drilling fluid leakage-proof and plugging materials. First, the rigid bridging particles build stable bridges at the positions of pore throat or narrow cracks and form the skeleton of the plugging layer; then the fiber materials and rigid particles act synergistically, which is helpful to form a dense network frame structure and improve the overall structural stability of the crack-plugging and pressure-bearing layer; after that, under the dual effects of squeeze deformation and elastic expansion, the elastic filling particles further fill in the pore spaces between the rigid bridging particles, and meanwhile they can adaptively plug the cracks. With the formation of the crack-plugging and pressure-bearing layer, the drilling fluid leakage loss gradually changes to filtration loss, and the asphalt-type and resin-type filtration loss reducing agents can further fill in the crack-plugging and pressure-bearing layer, and finally, a dense crack-plugging and pressure-bearing layer with a "strong-force chain network" is formed (Figure 9), which can significantly improve the plugging and pressure-bearing performance of the OBDF borehole wall, and it is helpful to improve the pressure-bearing ability of the earth stratums as well.

\section{CONCLUSIONS}

(1) Compared with WBDF, OBDF has the characteristics of "easy-to-leak and difficult-to-treat", therefore, it's necessary to further research the enhanced crack-plugging and pressurebearing OBDF system, and form a set of OBDF leakage-proof and plugging techniques and on-site supporting measures.

(2) In this study, a new-type OBDF drilling leakage-proof agent and a new-type OBDF still crack plugging agent were developed in laboratory; the proposed agents can significantly improve the crack-plugging and pressure-bearing performance of the OBDF borehole wall, and they are also helpful to improve the pressure-bearing ability of the easy-leaking earth stratums.

(3) Different types of drilling fluid leakage-proof and plugging materials such as rigid bridging particles, elastic filling particles, and fiber materials have a synergistic plugging effect, they can quickly form a dense crack-plugging layer with a "strong-force chain network", thus achieving the effect of borehole wall strengthening and plugging.

\section{REFERENCES}

[1] Wang, H., Sweatman, R.E., Engelman, R., Deeg, W.F., Whitfill, D.L., Soliman, M.Y., Towler, B.F. (2008). Best practice in understanding and managing lost circulation challenges. SPE Drilling \& Completion, 23(2): 168-175. https://doi.org/10.2118/95895-PA

[2] Hou, S.L., Huang, D.Q., Yang, H.W., Liu, G. (2015). Study and application of rigid wedging mud loss control under pressure. Drilling Fluid \& Completion Fluid, 32(1) 49-52. https://doi.org/10.3696/j.issn.10015620.2015 .01 .013

[3] Onyia, E.C. (1994). Experimental data analysis of lostcirculation problems during drilling with oil-based mud. SPE Drilling \& Completion, 9(1): 25-31. https://doi.org/10.2118/22581-PA

[4] Dupriest, F.E. (2005). Fracture closure stress (FCS) and lost returns practices. The SPE/IADC Drilling Conference, Amsterdam, Netherlands, SPE-92192-MS. https://doi.org/10.2118/92192-MS

[5] Benaissa, S., Bachelot, A., Ricaud, J., Arquey, G., Yi, X., Ong, S. (2005). Preventing differential sticking and mud losses drilling through highly depleted sands. fluids and geomechanics approach. The SPE/IADC Drilling Conference, Amsterdam, Netherlands, SPE-92266-MS. https://doi.org/10.2118/92266-MS

[6] Aderibigbe, A.A., Lane, R.H. (2013). Rock/fluid chemistry impacts on shale fracture behavior. The SPE International Symposium on Oilfield Chemistry, The Woodlands, Texas, USA, SPE-164102-MS. https://doi.org/10.2118/164102-MS

[7] Zhang, X.W., Li, S., Zhang, J., Sun, J. (2009). Research progress on lost circulation materials and lost circulation control technology. Drilling Fluid \& Completion Fluid, 26(6): 74-76.

[8] Chai, J., Liu, J., Qiu, Z. (2017). Experimental study on loss-prevention performance of oil-based drilling fluids. Advances in Petroleum Exploration and Development, 13(1), 70-77. https://doi.org/10.3968/9415

[9] Scorsone, J.T., Sanders, M.W., Patel, A.D. (2009). An improved oil-based chemical gel system for wellbore stabilization. the SPE International Symposium on Oilfield Chemistry, The Woodlands. Texas, SPE121676-MS. https://doi.org/10.2118/121676-MS

[10] Savari, S., Whiftill, D.L., Jamison, D.E., Kumar, A. (2014). A method to evaluate lost-circulation materialsinvestigation of effective wellbore-strengthening applications. SPE Drilling \& Completion, 29(3): 329333. https://doi.org/10.2118/167977-PA

[11] Wang, H., Soliman, M.Y., Towler, B.F., Mukai, D. (2008). Avoiding drilling problems by strengthening the wellbore while drilling. The 42nd U.S. Rock Mechanics Symposium (USRMS), San Francisco, California, ARMA-08-200

[12] Mostafavi, V., Hareland, G., Belayneh, M., Aadnoy, B.S. (2011). Experimental and mechanistic modeling of fracture sealing resistance with respect to fluid and fracture properties. The 45th U.S. Rock Mechanics / Geomechanics Symposium, San Francisco, California, ARMA-11-198.

[13] Kang, Y.L., Yu, H.F., Xu, C.Y., Tang, L. (2014). An optimal design for millimeter-wide fracture-plugged zones. Natural Gas Industry, 34(11): 88-94. https://doi.org/10.3787/j.issn.1000-0976.2014.11.012 